\title{
Impact of postoperative lymph node status on the prognosis of esophageal squamous cell carcinoma after esophagectomy following neoadjuvant chemoradiotherapy: a retrospective study
}

\author{
Zhiyong Sun ${ }^{1 \#}$, Xin $\mathrm{Xu}^{2 \#}$, Xiaojing Zhao ${ }^{1}$, Xiumei $\mathrm{Ma}^{2}$, Qing $\mathrm{Ye}^{1}$ \\ ${ }^{1}$ Department of Thoracic Surgery, Renji Hospital, School of Medicine, Shanghai Jiao Tong University, Shanghai, China; ${ }^{2}$ Department of Radiation \\ Oncology, Renji Hospital, School of Medicine, Shanghai Jiao Tong University, Shanghai, China \\ Contributions: (I) Conception and design: Q Ye, X Ma; (II) Administrative support: X Zhao; (III) Provision of study materials or patients: Z Sun, X \\ $\mathrm{Xu}$; (IV) Collection and assembly of data: Z Sun; (V) Data analysis and interpretation: Z Sun, X Xu; (VI) Manuscript writing: All authors; (VII) Final \\ approval of manuscript: All authors. \\ \#These authors contributed equally to this work. \\ Correspondence to: Qing Ye. Department of Thoracic Surgery, Renji Hospital, School of Medicine, Shanghai Jiao Tong University, 160 Pujian Road, \\ Shanghai 200127, China. Email: yeqing1310@renji.com; Xiumei Ma. Department of Radiation Oncology, Renji Hospital, School of Medicine, \\ Shanghai Jiao Tong University, 160 Pujian Road, Shanghai 200127, China. Email: sallyma@hotmail.com.
}

Background: Neoadjuvant chemoradiotherapy (nCRT) and surgery are widely used treatments for locally advanced esophageal squamous cell carcinoma (ESCC). Thus, it is critically important to investigate risk factors that affect patient prognosis after preoperative chemoradiotherapy and surgery.

Methods: We conducted a retrospective analysis of 77 patients with ESCC who received nCRT and underwent surgery at our center from January 2015 to December 2019. We analyzed the primary clinical data, postoperative pathological results, recurrence, and death results.

Results: Among the 77 ESCC patients who received nCRT and surgery, 19 achieved a postoperative pathologic complete response (pCR), and the overall pCR rate was $24.68 \%$. The univariate analysis indicated that postoperative post-neoadjuvant treatment $\mathrm{N}$ stage (ypN) metastasis [hazards ratio (HR): $2.908 ; 95 \%$ confidence interval (CI): 0.874-9.676; P=0.082], a high lymph-node ratio [(LNR) >0.1] (HR: 7.149, 95\% CI: 1.740-29.369; P=0.006), post-neoadjuvant treatment T3-4 (ypT3-4) (HR: 3.626, 95\% CI: 0.824-15.956; $\mathrm{P}=0.088)$ affected disease-specific survival (DSS). The multivariate analysis indicated that a high $\operatorname{LNR}(>0.1)$ (HR: 6.170; 95\% CI: 1.472-25.856; P=0.013) was a significant independent predictor of DSS. The univariate analysis indicated that postoperative ypN metastasis (HR: 2.283; 95\% CI: $1.047-4.979 ; \mathrm{P}=0.038$ ) and a high LNR (>0.1) (HR: 4.210; 95\% CI: 1.547-11.458; P=0.005) were associated with recurrence-free survival (RFS). The multivariate survival analysis showed that a high LNR (>0.1) (HR: 4.289; 95\% CI: 1.538-11.965; $\mathrm{P}=0.005$ ) was also a significant independent predictor of RFS. In this study, 57 positive lymph nodes were found in 30 of the 77 patients, including 16 left gastric lymph nodes, 9 pericardial lymph nodes, and 7 left supraclavicular lymph nodes.

Conclusions: A high LNR $(>0.1)$ in ESCC patients after nCRT is a risk factor of DSS and RFS. ypN metastasis is also an independent predictor of RFS. Left gastric-arterial lymph nodes, para-cardiac lymph nodes, and left supraclavicular lymph nodes are the most common sites of metastasis in ESCC after nCRT.

Keywords: Esophageal squamous cell carcinoma (ESCC); neoadjuvant chemoradiotherapy (nCRT); prognosis; lymph-node ratio (LNR); lymph nodes

Submitted Nov 05, 2021. Accepted for publication Dec 20, 2021.

doi: 10.21037/jgo-21-807

View this article at: https://dx.doi.org/10.21037/jgo-21-807

(c) Journal of Gastrointestinal Oncology. All rights reserved. 


\section{Introduction}

Surgery has been the mainstay of treatment for resectable esophageal squamous cell carcinoma (ESCC) for decades; however, the overall treatment outcome is poor, and the 5 -year survival rate is only $20.64-34.00 \%$ (1). In the CROSS trials, the pathologic complete response (pCR) rate of the neoadjuvant chemoradiotherapy (nCRT) group was $29 \%$, and the median overall survival (OS) of the nCRT group was significantly higher than that of the surgery group [48.6 vs. 24.0 months; hazards ratio (HR), 0.68; 95\% confidence interval (CI), $0.53-0.88 ; \mathrm{P}=0.003]$. Since only 84 patients (23\%) with ESCC were included in the study, the conclusions of the CROSS trials regarding ESCC remain controversial. The NEOCRTEC 5010 study in China confirmed that preoperative chemoradiotherapy could significantly increase the survival of patients with locally advanced ESCC; however, the recurrence rate of ESCC after nCRT is $>40 \%$ (2). Thus, it is essential to explore the recurrence factors of ESCC after nCRT.

We present the following article in accordance with the STROBE reporting checklist (available at https://dx.doi. org/10.21037/jgo-21-807).

\section{Methods}

\section{Patient population}

A retrospective study was conducted. The data of 103 patients with locally advanced ESCC who received nCRT from January 2015 to December 2019 were collected. Twenty-three final non-surgical patients were excluded (including 10 patients who refused surgery after treatment, and 13 patients with disease progression). In addition, 3 patients who were lost during the follow-up period after surgery were excluded from the study. Thus ultimately, 77 patients (70 male and 7 female) were included in the study (see Figure 1). The patients had an average age of 62.5 years (range, 48-74 years).

All procedures involving human participants conducted in this study conformed to the Helsinki Declaration (as revised in 2013). This study was approved by the Ethics Committee of the Renji Hospital, Shanghai Jiaotong University School of Medicine with approval number KY2020-032. As this was a retrospective study, informed consent was not required.

\section{Preoperative evaluation methods}

After being diagnosed with ESCC by gastroscopy before treatment, further chest enhanced computed tomography (CT), a cervical lymph-node ultrasound, and an abdominal ultrasound were performed. A positron emission tomography (PET)-CT scan was also performed in most cases. The complete pulmonary function test and cardiac ultrasound evaluation were performed at the same time. After a multidisciplinary team discussion, preoperative chemoradiotherapy was administered to patients with clinical stage cT3-4N0M0 or cT1-4N1-2M0.

\section{NCRT}

All patients received paclitaxel combined with cisplatin, and radiation of $41.4 \mathrm{~Gy} / 23$ fractions that complied with intensity-modulated radiotherapy or volumetric-modulated arc therapy. 1 of the following 2 chemotherapy regimens was selected and administered: (I) a 2-weekly regimen of paclitaxel $\left(135 \mathrm{mg} / \mathrm{m}^{2}\right)+$ cisplatin $\left(75 \mathrm{mg} / \mathrm{m}^{2}\right)$ every 3 weeks for 2 cycles; or (II) a weekly regimen of paclitaxel $\left(45 \mathrm{mg} / \mathrm{m}^{2}\right)+$ cisplatin $\left(25 \mathrm{mg} / \mathrm{m}^{2}\right)$ every 2 weeks for 5 cycles.

\section{Operation time and plan}

Clinical restaging was performed approximately 4-6 weeks after chemoradiotherapy. Patients without disease progression were scheduled for surgery, and patients with disease progression continued chemoradiotherapy. Surgery was performed 6-8 weeks after chemoradiotherapy. A minimally invasive McKeown or Ivor Lewis esophagectomy was performed.

\section{Postoperative follow-up and data collection}

We analyzed data about overall characteristics, complications, local recurrence, distant metastasis, survival time, and other related data. The preoperative and postoperative pathological stages, and the results of the patients were recorded. All of the patients were regularly followed-up at outpatient clinic appointments or by telephone. All the data were confirmed by two doctors to avoid any bias caused by the data collection and input deviation.

\section{Statistical analysis}

The statistical analysis was performed using SPSS 26.0. The Kaplan-Meier method was used to draw the survival 


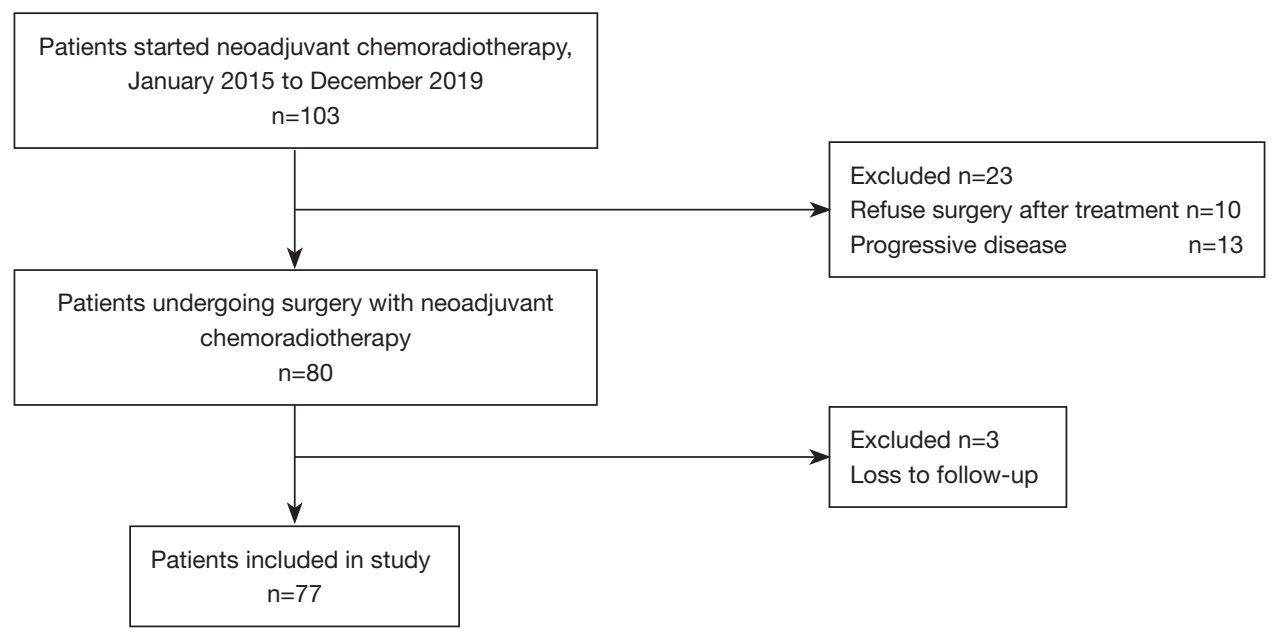

Figure 1 Study flow chart.

curves, and the log-rank method was used to compare the differences between the survival curves. In the univariate analysis, the Kaplan-Meier method was used, and factors with a $\mathrm{P}$ value $<0.1$ as tested by the log-rank method were included in the Cox proportional risk model to evaluate the independent prognostic factors. Recurrence-free survival (RFS) was defined as the time between the initial operation and the earliest evidence of recurrence. Disease-specific survival (DSS) was defined as any patient in the study or treatment group did not die from a specific disease during the defined period. A $\mathrm{P}$ value $<0.05$ indicated a statistically significant difference.

\section{Results}

\section{Overall characteristics}

Seventy-seven patients (70 male and 7 female) who received nCRT and underwent minimally invasive esophagectomy from January 2015 to December 2019 were included in the study. The patients had an average age of 62.5 years (range, 48-74 years); 23 patients were aged $\leq 60$ years and 54 patients were aged $>60$ years. The tumors were located in the proximal third, middle third, and distal third in 7, 33, and 37 cases, respectively. In relation to preoperative clinical staging, there were $61(79.2 \%)$ cT3 cases and $12(15.6 \%)$ cT4 cases. There were 42 (54.5\%) cases of cN1, 30 (39\%) cases of $\mathrm{cN} 2$, and $1(1.3 \%)$ case of $\mathrm{cN} 3$. Postoperatively, there were $18(18.2 \%)$ cases of ypT3, 5 (6.5\%) cases of ypN2, and 1 (1.3\%) case of ypN3.

Postoperative pCR was achieved in 19 (24.68\%) cases. In
$61(79.2 \%)$ cases, $>15$ lymph nodes were dissected during surgery (range, 15-53; average 24.21). Twelve patients had anastomotic complications, including anastomotic leakage in $10(12.98 \%)$ cases, anastomotic bleeding in 2 cases, postoperative intestinal obstruction in 1 case, pulmonary embolism in 1 case, and heart failure in 1 case (see Table 1 ).

\section{Univariate and multivariate analysis}

The univariate analysis showed that postoperative ypN metastasis (HR: 2.283; 95\% CI: 1.047-4.979; $\mathrm{P}=0.038$ ), and a high lymph-node ratio [(LNR) >0.1] (HR: 4.210; 95\% CI: $1.5477-11.458 ; \mathrm{P}=0.005)$ were associated with the RFS of patients after esophagectomy following nCRT. The multivariate Cox regression analysis showed that a high LNR (>0.1) (HR: 4.289; 95\% CI: 1.5388-11.965; P=0.005) was a significant independent predictor of RFS (see Table 2).

In this study, 6 ESCC patients died of unrelated diseases ( 2 of sudden cardiac death at home, 2 of cerebrovascular accidents, 1 of leukemia, and 1 of epilepsy). Thus, DSS was taken as the endpoint of this study. The univariate analysis indicated that postoperative ypN metastasis (HR: 2.908; 95\% CI: 0.874-9.676; $\mathrm{P}=0.082)$, a high LNR $(>0.1)$ (HR: 7.149; 95\% CI: 1.740-29.369; P=0.006), ypT34 (HR: 3.626; 95\% CI: 0.824-15.956; $\mathrm{P}=0.088$ ) possibly affected DSS. The multivariate analysis indicated that a high postoperative LNR (>0.1) (HR: 6.170; 95\% CI: $1.472-$ 25.856; $\mathrm{P}=0.013$ ) was an independent prognostic factor of DSS (see Table 3). Due to the strong linear relationship between the ypN status and LNR, only LNR was included in the multivariate analysis. 
Table 1 Patient characteristics $(\mathrm{n}=77)$

\begin{tabular}{|c|c|}
\hline Variable & Value \\
\hline \multicolumn{2}{|l|}{ Age $(\%)$} \\
\hline$\leq 60$ years & $23(29.8)$ \\
\hline$>60$ years & $54(70.2)$ \\
\hline \multicolumn{2}{|l|}{$\operatorname{Sex}(\%)$} \\
\hline Male & 70 (90.9) \\
\hline Female & $7(9.1)$ \\
\hline \multicolumn{2}{|l|}{ Clinical T category (\%) } \\
\hline cT1 & $1(1.3)$ \\
\hline сT2 & $3(3.9)$ \\
\hline сT3 & $61(79.2)$ \\
\hline cT4 & $12(15.6)$ \\
\hline \multicolumn{2}{|l|}{ Clinical N category (\%) } \\
\hline cNO & $4(5.2)$ \\
\hline $\mathrm{cN} 1$ & $42(54.5)$ \\
\hline $\mathrm{cN} 2$ & $30(39.0)$ \\
\hline cN3 & $1(1.3)$ \\
\hline \multicolumn{2}{|l|}{ Pathological T category (\%) } \\
\hline урто & $28(36.4)$ \\
\hline ypT1 & $17(22.1)$ \\
\hline урт2 & $14(23.3)$ \\
\hline урт3 & $18(18.2)$ \\
\hline ypT4 & $0(0)$ \\
\hline \multicolumn{2}{|l|}{ Pathological $\mathrm{N}$ category (\%) } \\
\hline ypNO & $47(61.0)$ \\
\hline ypN1 & $24(31.2)$ \\
\hline ypN2 & $5(6.5)$ \\
\hline ypN3 & $1(1.3)$ \\
\hline \multicolumn{2}{|l|}{ Tumor location (\%) } \\
\hline Upper & $7(9.1)$ \\
\hline Middle & $33(42.9)$ \\
\hline Lower & $37(48.0)$ \\
\hline \multicolumn{2}{|l|}{ LN dissection number (\%) } \\
\hline$<15$ & $16(20.8)$ \\
\hline$\geq 15$ & $61(79.2)$ \\
\hline \multicolumn{2}{|l|}{ Postoperative complications (\%) } \\
\hline Anastomotic complications & $12(15.6)$ \\
\hline Cardiac complications & $1(1.3)$ \\
\hline Bowel obstruction & $1(1.3)$ \\
\hline Pulmonary complications & $1(1.3)$ \\
\hline Others & $3(3.9)$ \\
\hline
\end{tabular}

\section{Survival curves for DSS and RFS}

Patients were divided into (three groups based on their LNR: (I) the LNR 0 group (LNR =0); (II) the low LNR group $(0<\mathrm{LNR} \leq 0.1)$; and (III) the high LNR group (LNR $>0.1)$. The survival analysis revealed a substantial difference in the DSS $(\mathrm{P}=0.007)$ and RFS $(\mathrm{P}=0.010)$ among the LNR groups (see Figure 2A,2B). The 1- and 3-year DSS of the LNR 0 group, the low LNR group, and the high LNR group were $100 \%$ and $96.2 \%, 90.9 \%$ and $84.8 \%$, and $75 \%$ and $0 \%$, respectively. The data of the three groups were statistically different $(\mathrm{P}=0.007)$.

When RFS was compared between the two different ypN statuses, the prognosis of the ypN+ group was poor, and the difference was significant $(\mathrm{P}=0.031)$. Of the 47 patients with ypN0, 11 had recurrence at follow-up, and 15 of the 30 patients with ypN+ had a recurrence. The $1-$ and 3 -year RFS of the two groups were $82.1 \%$ and $68.4 \%$, and $66.7 \%$ and $48.9 \%$, respectively, but there was no statistical difference in DSS between the groups $(\mathrm{P}=0.067)$ (see Figure 2C,2D).

\section{Distribution of lymph node metastasis after nCRT}

After the operation, the lymph nodes were separated and marked, and immediately fixed with formalin. Two pathologists evaluated them separately. The problematic cases were identified by immunohistochemically stained serial sections. Among the 77 patients, 30 had lymph node metastasis after surgery, and a total of 57 lymph nodes were dissected. Among them, 16 metastases were found in the left gastric-arterial lymph nodes (No. 7), 9 metastases were found in the para-cardiac lymph nodes (No. $1+$ No. 2), 7 metastases were found in the left supraclavicular lymph nodes (No. 104L), and 6 metastases were found in the subcarinal lymph nodes (No. 107) (see Figure 3).

\section{Discussion}

Esophageal cancer is the eighth most common cancer type and the sixth leading cause of cancer deaths in the world. It is characterized by its high mortality rate, poor prognosis at time of diagnosis and variability based on geographic location. Esophageal squamous cell carcinoma continues to be the most prevalent type worldwide (3). However, mortality has declined with the improvement of the treatment model. At present, the 5 -year OS is $30-57 \%$ (4).

ESCC is the most common histology in Asia and Eastern 
Table 2 Univariable and multivariable analyses of RFS

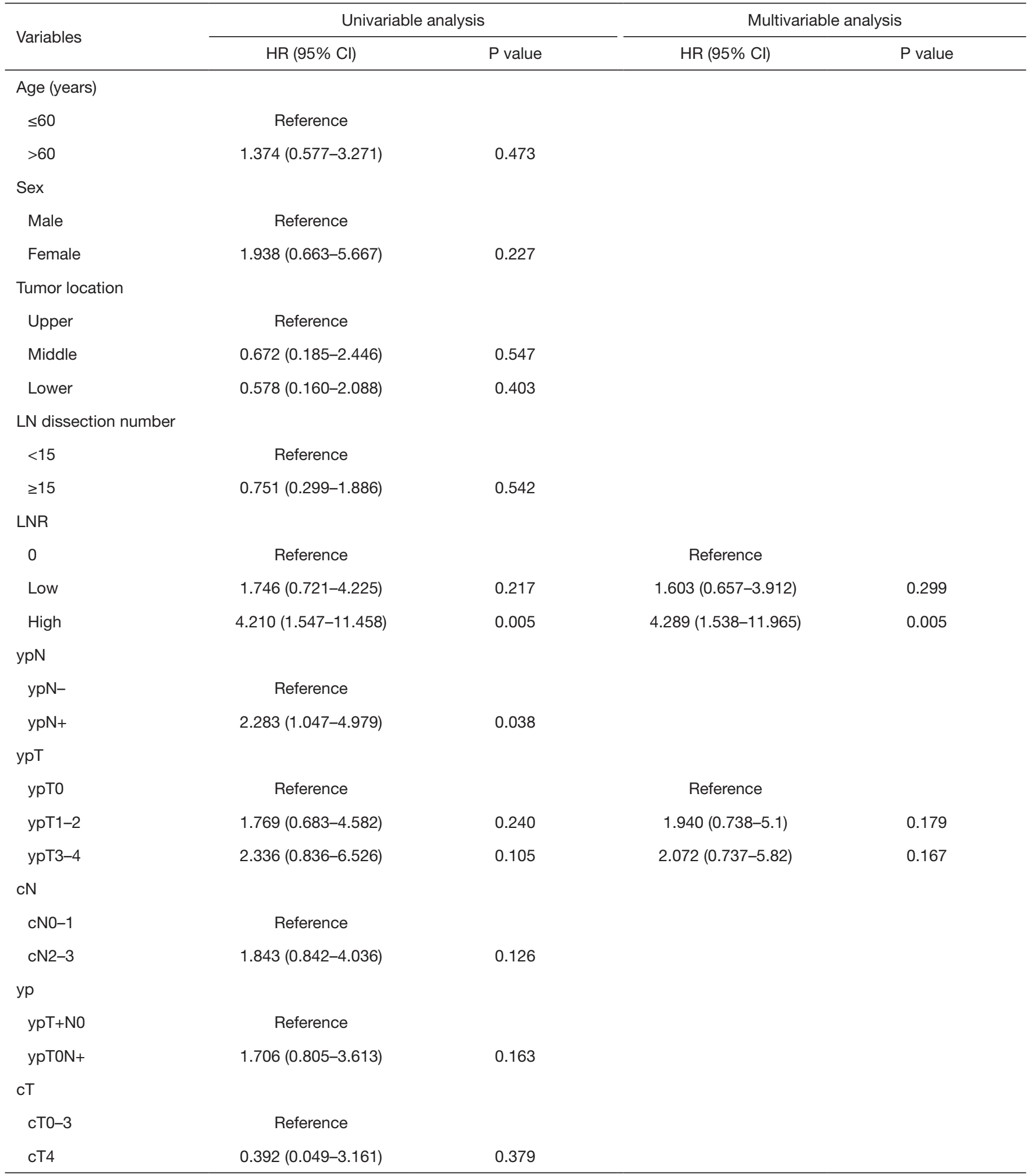

RFS, recurrence-free survival. 
Table 3 Univariable and multivariable analyses of DFS

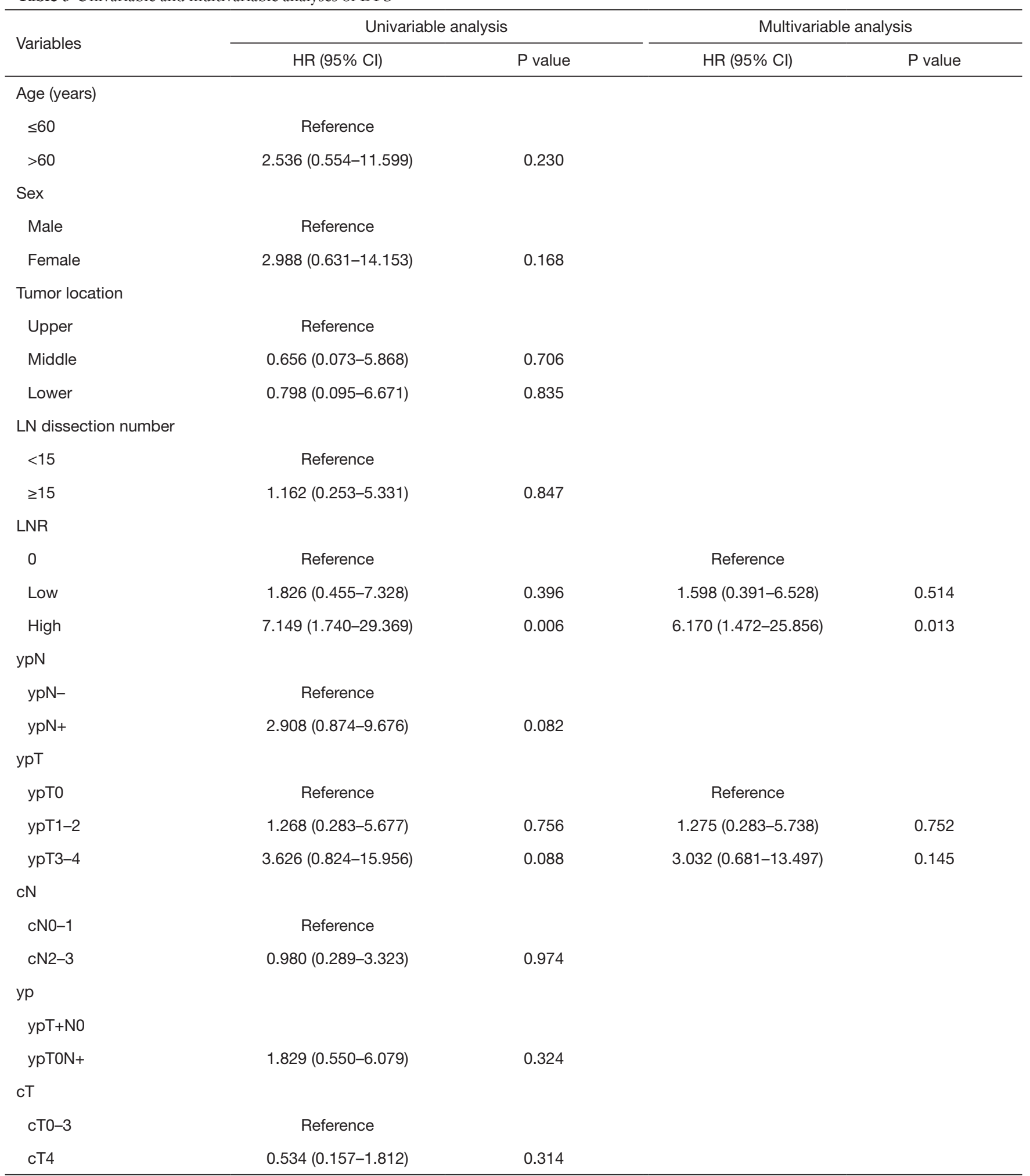

DFS, disease-free survival. 
A

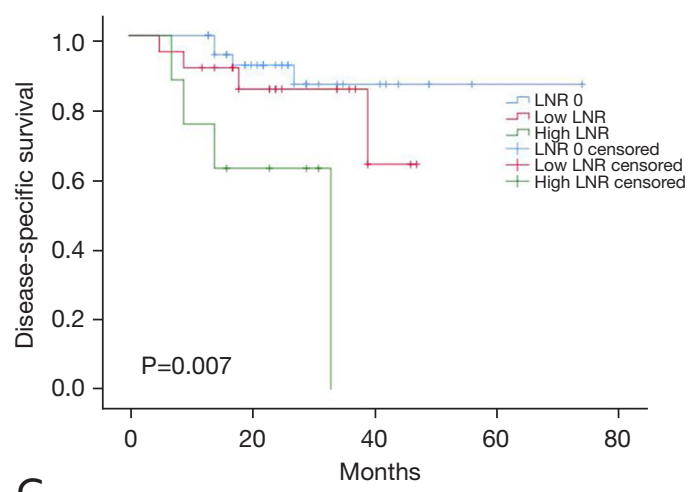

C

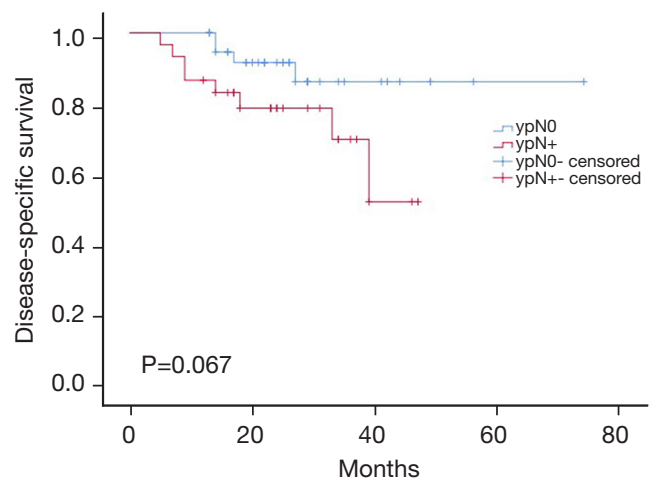

B

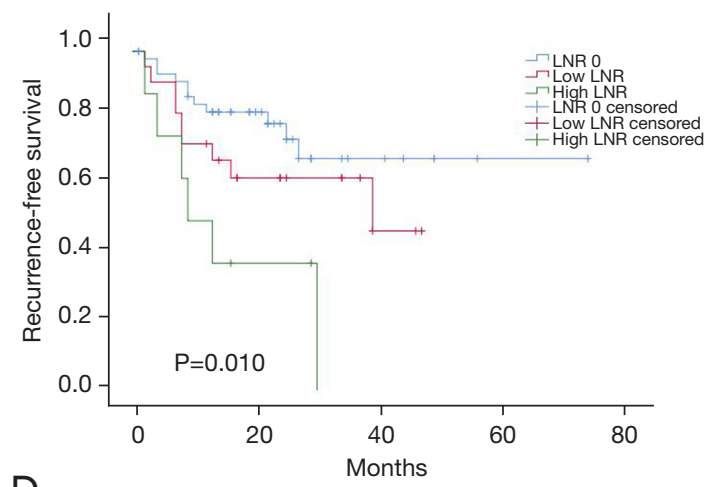

D

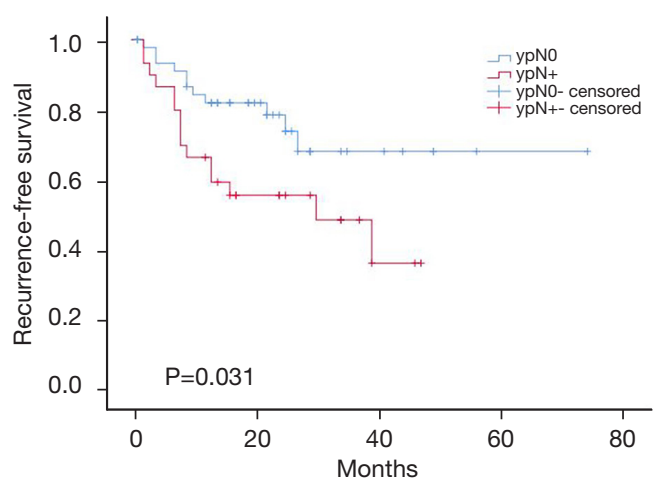

Figure 2 DFS and RFS of ESCC after nCRT. (A,B) Curves of DFS and RFS with different LNR after nCRT plus surgery; (C,D) curves of DFS and RFS with ypN status after nCRT plus surgery. DFS, disease-free survival; RFS, recurrence-free survival; ESCC, esophageal squamous cell carcinoma; nCRT, neoadjuvant chemoradiotherapy; LNR, lymph-node ratio.

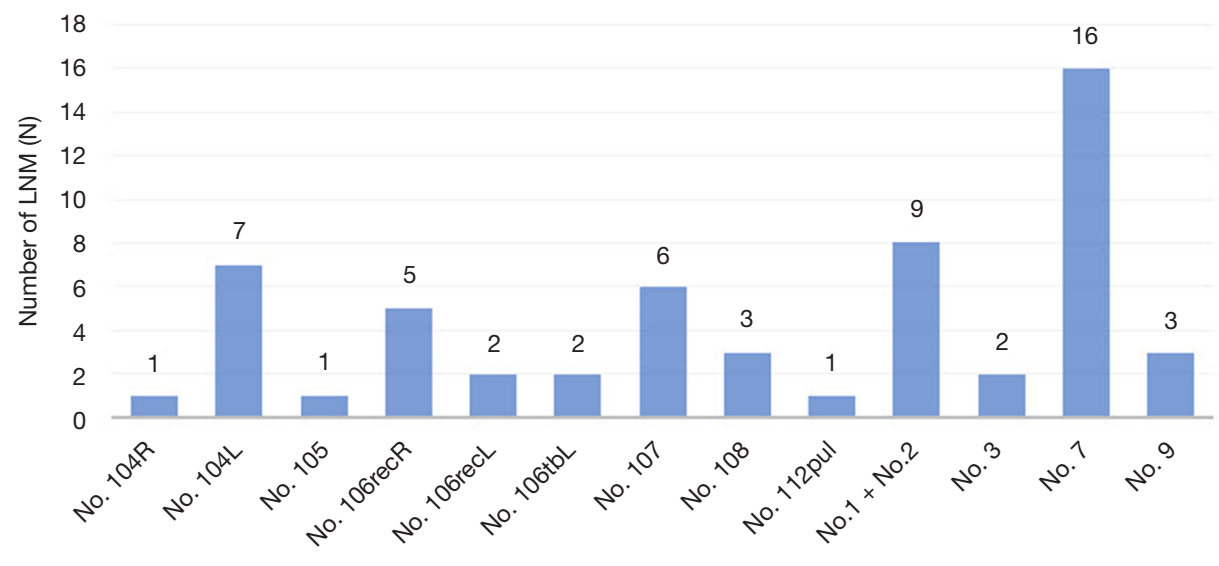

LN stations

Figure $3 \mathrm{ypN}$ metastasis in 30/77 patients. 
Europe, while adenocarcinoma accounts for $>70 \%$ of all esophageal cancers in the United States and Western Europe (5). Most patients are in an advanced stage at the time of their treatment, so treatment outcomes are often unsatisfactory. This study mainly focused on ESCC after nCRT.

The 2002 CROSS trials showed that both the OS and disease-free survival (DFS) of patients in the nCRT group were significantly longer than the OS and DFS of patients in the surgery group, and the differences were statistically significant $(\mathrm{P}<0.05)(6)$. The results of the NEOCRTEC 5010 study in China showed that patients who received a combination of nCRT and surgery had a better OS group than those who received surgery group (median OS: 100.1 vs. 66.5 ; HR, $0.71 ; 95 \%$ CI: 0.53 to $0.96 ; \mathrm{P}=0.025)$ and had prolonged DFS (median DFS: 100.1 vs. 41.7 months; HR, 0.58 ; $95 \%$ CI: 0.43 to 0.78 ; $\mathrm{P}<0.001)(7)$. Thus, to reduce pathological lymph-node metastasis and local recurrence, treating esophageal cancer patients with NCRT is more effective than treating patients with surgery alone (8). nCRT has been increasingly accepted as the standard of treatment, as reflected by the latest National Comprehensive Cancer Network (NCCN) guidelines (9).

For the patients included in this study, the neoadjuvant treatment regimen also followed the recommendations of the CROSS trials, and the chemotherapy regimen comprised carboplatin combined with paclitaxel. A total radiation dose of 41.4 Gy was given in 23 fractions of 1.8 Gy each, with 5 fractions administered per week. Several studies have confirmed that pCR remains one of the most important prognostic factors of long-term survival in ESCC after nCRT followed by surgery $(10,11)$. In our study, the total number of pCR cases was 19 (24.67\%), of which 3 cases ( 2 cases of bone metastasis and 1 case of liver metastasis) were found to have tumor progression during follow-up. Xi found that the pCR rate was significantly higher in ESCC patients than adenocarcinoma patients (44.9\% vs. $25.9 \% ; \mathrm{P}<0.001)$ (12). The $\mathrm{pCR}$ rate of this study was generally lower than that of the above research. Thus, further research needs to be conducted.

Some experts believe that the ypT category, tumor grade in lymph-node metastasis, and the number of lymphnode metastases may be significant independent predictors of DFS and OS (13). The LNR (i.e., the ratio of the number of positive lymph nodes to the total number of removed lymph nodes) has gained attention as an important prognostic factor in gastrointestinal cancer and breast cancer (14). However, very few studies on the application of LNR after neoadjuvant therapy for ESCC. Bollschweiler et al. (15) found a significant difference in patient survival when the LNR was $>0.2(\mathrm{P}<0.01)$. Nigro et al. $(16)$ found a significant difference in patient survival when the LNR was 0.1 . Based on the average dissection of 24.09 lymph nodes per case in this study, N1 and N2 were defined by the NCCN guidelines, and 0.1 was selected as the cutoff value of LNR. In our study, the univariate and multivariate analyses suggested that a high LNR $(>0.1)$ might affect DSS (HR: 6.170, 95\% CI: 1.472-25.856; $\mathrm{P}=0.013$ ) and RFS (HR: 4.289, 95\% CI: 1.5388-11.965; $\mathrm{P}=0.005$ ). In the univariate analysis, in addition to the LNR, the ypN status was significantly correlated with DSS (HR: 2.908, 95\% CI: 0.874-9.676; $\mathrm{P}=0.082$ ) and RFS (HR: 2.283 , 95\% CI: 1.047-4.979; $\mathrm{P}=0.038$ ). Due to the strong linear relationship between the ypN status and the LNR, only the LNR was included in the multivariate analysis.

According to the survival curves for DSS and RFS, we found that there was no statistically significant difference in DSS $(\mathrm{P}=0.067)$ between the ypN+ and ypN0 cases, but there was a statistically significant difference in RFS between the ypN+ and ypN0 cases $(\mathrm{P}=0.031)$. The LNR was used to allocate patients into three groups. The survival analysis indicated a substantial difference in DSS $(\mathrm{P}=0.007)$ and RFS $(\mathrm{P}=0.010)$ among the LNR groups. Thus, a high LNR appears to be an independent risk factor of RFS and DSS, and ypN metastasis is an independent predictor of RFS but not DSS.

The results of the NEOCRTEC 5010 study showed that the OS and DFS of patients with negative postoperative lymph nodes in the nCRT group and the surgery group were higher than those in the groups of positive postoperative lymph nodes. The partial remission of the primary tumor (HR 2.09) and negative lymph nodes (HR 3.26) were independent risk factors of DFS but not OS. The recurrence rate in patients who obtained pCR was $13.9 \%$ (17). Thus, we believe that persistent pathological positive lymph nodes after nCRT is a high-risk factor for the recurrence of ESCC, and a high LNR may indicate a poor long-term prognosis after surgery. pCR does not mean a cure, and close postoperative follow-up is also very important.

The status of lymph nodes has been considered the most important prognostic factor affecting the long-term survival of ESCC patients. In this study, 30 patients had lymph node metastasis after surgery. A total of 57 lymph nodes were dissected; 16 gastric-arterial lymph nodes (No. 7), 9 gastric paracardial lymph nodes (No. $1+$ 
No. 2), 7 left supraclavicular lymph nodes (No. 104L), and 6 subcarinal lymph nodes (No. 107). These areas remain the most common metastatic areas for esophageal cancer.

Radical lymphadenectomy might be an important method for improving survival (9). The dissection of lymph nodes could be helpful in the accurate pathological staging of esophageal cancer after surgery, ensure the radical treatment of tumors, and enable the outcome of neoadjuvant therapy to be evaluated. Shi (18) believes that for patients with a higher $\mathrm{T}$ stage, at least 17 lymph nodes should be dissected. As for the standard of lymph-node dissection for ESCC after nCRT, more reliable clinical data need to be gathered.

According to our study, for ESCC cases after nCRT, if there is lymph node metastasis or LNR is greater than 0.1 after operation, we should be alert to the possibility of postoperative recurrence. Standardized lymph node dissection during surgery will allow us to obtain a more reliable clinical result.

This study had some limitations. As this is a retrospective analysis, some variables that might affect the survival rate were missing from the database and could not be integrated into the Cox regression models. The current commonly used clinical examination and evaluation methods are inaccurate at evaluating tumor tissue responses after nCRT. We prefer using PET/CT combined with chest enhanced CT to evaluate lymph node metastasis. Unfortunately, it is still difficult to accurately diagnose lymph node status using the available diagnostic systems. CT scans are widely used for the nodal staging of esophageal cancer. However, several studies have found that the sensitivity and specificity of CT scans are low (19). The diagnosis of lymph nodes, especially in the paracardial (Nos. 1 and 2) and paratracheal (No. 106) regions, need to be considered carefully, as these are the areas in which false-negative lymph nodes are most found (20). As for PET/CT, it is unable to detect microscopic metastasis and differentiate nodal metastatic disease from inflammation, but it is very useful at detecting distant metastases (21). Some experts have found that a composite CT/PET radiomics model is highly predictive of pCR following nCRT (22). Developing a reliable predictive method could optimize treatment through the accurate selection of patients.

\section{Conclusions}

A high LNR (>0.1) for ESCC after nCRT is a risk factor of DSS and RFS. ypN metastasis is also an independent predictor of RFS. Left gastric-arterial lymph nodes, paracardiac lymph nodes, and left supraclavicular lymph nodes are still the most common sites of metastasis for esophageal cancer after nCRT.

\section{Acknowledgments}

We would like to thank all the members of the Department of Thoracic Surgery and the Department of Radiation Oncology at our hospital who participated in this research. Funding: None.

\section{Footnote}

Reporting Checklist: The authors have completed the STROBE reporting checklist. Available at https://dx.doi. org/10.21037/jgo-21-807

Data Sharing Statement: Available at https://dx.doi. org/10.21037/jgo-21-807

Conflicts of Interest: All authors have completed the ICMJE uniform disclosure form (available at https://dx.doi. org/10.21037/jgo-21-807). The authors have no conflicts of interest to declare.

Ethical Statement: The authors are accountable for all aspects of the work in ensuring that questions related to the accuracy or integrity of any part of the work are appropriately investigated and resolved. All procedures involving human participants conducted in this study conformed to the Helsinki Declaration (as revised in 2013). This study was approved by the Ethics Committee of the Renji Hospital, Shanghai Jiaotong University School of Medicine with approval number KY2020-032. As this was a retrospective study, informed consent was not required.

Open Access Statement: This is an Open Access article distributed in accordance with the Creative Commons Attribution-NonCommercial-NoDerivs 4.0 International License (CC BY-NC-ND 4.0), which permits the noncommercial replication and distribution of the article with the strict proviso that no changes or edits are made and the original work is properly cited (including links to both the formal publication through the relevant DOI and the license). See: https://creativecommons.org/licenses/by-nc-nd/4.0/. 


\section{References}

1. de Jongh M, Eyck BM, van der Werf LR, et al. Pattern of recurrence in patients with a pathologically complete response after neoadjuvant chemoradiotherapy and surgery for oesophageal cancer. BJS Open 2021;5:zrab022.

2. Guo X, Wang Z, Yang H, et al. Impact of Lymph Node Dissection on Survival after Neoadjuvant Chemoradiotherapy for Locally Advanced Esophageal Squamous Cell Carcinoma: From the Results of NEOCRTEC5010, a Randomized Multicenter Study. Ann Surg 2021. [Epub ahead of print].

3. Shang QX, Yang YS, Hu WP, et al. Clinical and prognostic significance of preoperative lymphocyte-monocyte ratio, neutrophil-lymphocyte ratio and neutrophil-monocyte ratio on esophageal squamous cell carcinoma patients. Transl Cancer Res 2020;9:3903-14.

4. Buderi SI, Shackcloth M, Page RD. Does neoadjuvant chemoradiotherapy increase survival in patients with resectable oesophageal cancer? Interact Cardiovasc Thorac Surg 2017;24:115-20.

5. Arnold M, Laversanne M, Brown LM, et al. Predicting the Future Burden of Esophageal Cancer by Histological Subtype: International Trends in Incidence up to 2030. Am J Gastroenterol 2017;112:1247-55.

6. van Hagen P, Hulshof MC, van Lanschot JJ, et al. Preoperative chemoradiotherapy for esophageal or junctional cancer. N Engl J Med 2012;366:2074-84.

7. Yang H, Liu H, Chen Y, et al. Neoadjuvant Chemoradiotherapy Followed by Surgery Versus Surgery Alone for Locally Advanced Squamous Cell Carcinoma of the Esophagus (NEOCRTEC5010): A Phase III Multicenter, Randomized, Open-Label Clinical Trial. J Clin Oncol 2018;36:2796-803.

8. Oppedijk V, van der Gaast A, van Lanschot JJ, et al. Patterns of recurrence after surgery alone versus preoperative chemoradiotherapy and surgery in the CROSS trials. J Clin Oncol 2014;32:385-91.

9. National Comprehensive Cancer Network. Esophagus: version 3. 2015. Available online: http://www.nccn.org/ professionals/physician_gls/pdf/esophageal.pdf. Published 2015. Accessed November 24, 2015.

10. Soror T, Kho G, Zhao KL, et al. Impact of pathological complete response following neoadjuvant chemoradiotherapy in esophageal cancer. J Thorac Dis 2018;10:4069-76.

11. van Hagen $\mathrm{P}$, Wijnhoven BP, Nafteux P, et al. Recurrence pattern in patients with a pathologically complete response after neoadjuvant chemoradiotherapy and surgery for oesophageal cancer. Br J Surg 2013;100:267-73.

12. Xi M, Yang $Y$, Zhang L, et al. Multi-institutional Analysis of Recurrence and Survival After Neoadjuvant Chemoradiotherapy of Esophageal Cancer: Impact of Histology on Recurrence Patterns and Outcomes. Ann Surg 2019;269:663-70.

13. Van De Voorde L, Larue RT, Pijls M, et al. A qualitative synthesis of the evidence behind elective lymph node irradiation in oesophageal cancer. Radiother Oncol 2014;113:166-74.

14. Marchet A, Mocellin S, Ambrosi A, et al. The ratio between metastatic and examined lymph nodes ( $\mathrm{N}$ ratio) is an independent prognostic factor in gastric cancer regardless of the type of lymphadenectomy: results from an Italian multicentric study in 1853 patients. Ann Surg 2007;245:543-52.

15. Bollschweiler E, Baldus SE, Schröder W, et al. Staging of esophageal carcinoma: length of tumor and number of involved regional lymph nodes. Are these independent prognostic factors? J Surg Oncol 2006;94:355-63.

16. Nigro JJ, DeMeester SR, Hagen JA, et al. Node status in transmural esophageal adenocarcinoma and outcome after en bloc esophagectomy. J Thorac Cardiovasc Surg 1999;117:960-8.

17. Leng $X, H e W$, Yang $H$, et al. Prognostic Impact of Postoperative Lymph Node Metastases After Neoadjuvant Chemoradiotherapy for Locally Advanced Squamous Cell Carcinoma of Esophagus: From the Results of NEOCRTEC5010, a Randomized Multicenter Study. Ann Surg 2021;274:e1022-9.

18. Shi Y, Xu J, Wang Y, et al. Prognostic significance of preoperative lymph node assessment for patients with stage $\mathrm{pN} 0$ esophageal squamous cell carcinoma after esophagectomy. J Thorac Dis 2019;11:732-43.

19. Liu J, Wang Z, Shao H, et al. Improving CT detection sensitivity for nodal metastases in oesophageal cancer with combination of smaller size and lymph node axial ratio. Eur Radiol. 2018;28:188-95.

20. Wakita A, Motoyama S, Sato Y, et al. Evaluation of metastatic lymph nodes in cN0 thoracic esophageal cancer patients with inconsistent pathological lymph node diagnosis. World J Surg Oncol 2020;18:111.

21. Kim JJ, Park JK, Moon SW. Usefulness of positron emission tomography-computed tomography in preoperative evaluation of intra-thoracic esophageal cancer. Thorac Cancer 2015;6:687-94. 
22. Rishi A, Zhang GG, Yuan Z, et al. Pretreatment CT and 18 F-FDG PET-based radiomic model predicting pathological complete response and loco-regional control following neoadjuvant chemoradiation in oesophageal

Cite this article as: Sun Z, Xu X, Zhao X, Ma X, Ye Q. Impact of postoperative lymph node status on the prognosis of esophageal squamous cell carcinoma after esophagectomy following neoadjuvant chemoradiotherapy: a retrospective study. J Gastrointest Oncol 2021;12(6):2685-2695. doi: 10.21037/jgo-21-807 cancer. J Med Imaging Radiat Oncol 2021;65:102-11.

(English Language Editor: L. Huleatt) 